Mappemonde

Revue trimestrielle sur l'image géographique et les formes du territoire

$125 \mid 2019$

Varia

\title{
Le Champ-de-Mars, un « haut lieu » sportif ?
}

Hugo Bourbillères, Barbara Evrard et Dominique Charrier

\section{OpenEdition}

\section{Journals}

Édition électronique

URL : http://journals.openedition.org/mappemonde/780

DOI : 10.4000/mappemonde.780

ISSN : 1769-7298

Éditeur

UMR ESPACE

\section{Référence électronique}

Hugo Bourbillères, Barbara Evrard et Dominique Charrier, « Le Champ-de-Mars, un « haut lieu » sportif? », Mappemonde [En ligne], 125 | 2019, mis en ligne le 01 janvier 2019, consulté le 15 septembre 2020. URL : http://journals.openedition.org/mappemonde/780

Ce document a été généré automatiquement le 15 septembre 2020

\section{cc) (i) (2) (2)}

La revue Mappemonde est mise à disposition selon les termes de la Licence Creative Commons Attribution - Pas d'Utilisation Commerciale - Partage dans les Mêmes Conditions 4.0 International. 


\title{
Le Champ-de-Mars, un « haut lieu » sportif?
}

\author{
Hugo Bourbillères, Barbara Evrard et Dominique Charrier
}

1 Ouvert en $1780^{1}$, le parc du Champ-de-Mars s'étend entre l'École militaire et la tour Eiffel dans le $7^{\mathrm{e}}$ arrondissement de Paris. Haut lieu de grandes manifestations nationales et de tourisme (plus de 7 millions de visiteurs par an), il est en accès libre et offre la plus belle perspective sur le monument phare de la capitale. Avec ses 24,5 hectares, il est aussi l'un des plus grands espaces verts parisiens. Dans un contexte de densité urbaine et de saturation des équipements sportifs, il est donc assez logiquement le lieu de nombreuses manifestations sportives (Bourbillères, 2017).

2 Les événements qui s'y tiennent comportent par essence une ouverture vers l'extérieur liée à la gratuité ou au concept de l'événement. En conséquence, se démarquer parmi l'immensité diluante des espaces parisiens est un enjeu central. Par l'utilisation d'attributs matériels ou immatériels, ils construisent leur identité en ayant recours à une sémiotique patrimoniale. La course à pied «La Parisienne» et le dispositif «Roland Garros dans la ville » en sont l'illustration.

\section{Le marquage dynamique de « La Parisienne » : un Paris jeune et festif}

«La Parisienne » est une course féminine organisée autour du Champ-de-Mars chaque année au mois de septembre par l'entreprise du même nom. Cet événement créé en 1997 cristallise un positionnement singulier qui trouve l'assentiment du public (plus de 40000 participantes en 2017). Ce positionnement à la fois festif et dynamique surfe sur une approche ludique du sport, épousant certaines valeurs sociétales dont il peut se faire l'écho (santé, détente, « performance au quotidien »).

4 Le marquage urbain fait partie intégrante de l'expérience promise par les organisateurs. Sur site, il permet concrètement de transformer un lieu touristique mondialement connu en un espace de fête et de bonhomie autour de la pratique 
sportive féminine. De manière générale, "La Parisienne " est très colorée par l'utilisation de nombreuses oriflammes et banderoles disposées autour de la place de l'École militaire (village partenaire) et du parcours. En raison du positionnement dynamique de l'événement, les couleurs fluo s'observent aussi sur les tenues des participantes ou des bénévoles consacrant un marquage présence (Veschambre, 2007).

Figure 1. Les couleurs très vives s'inscrivent dans l'ambiance festive générale de l'événement

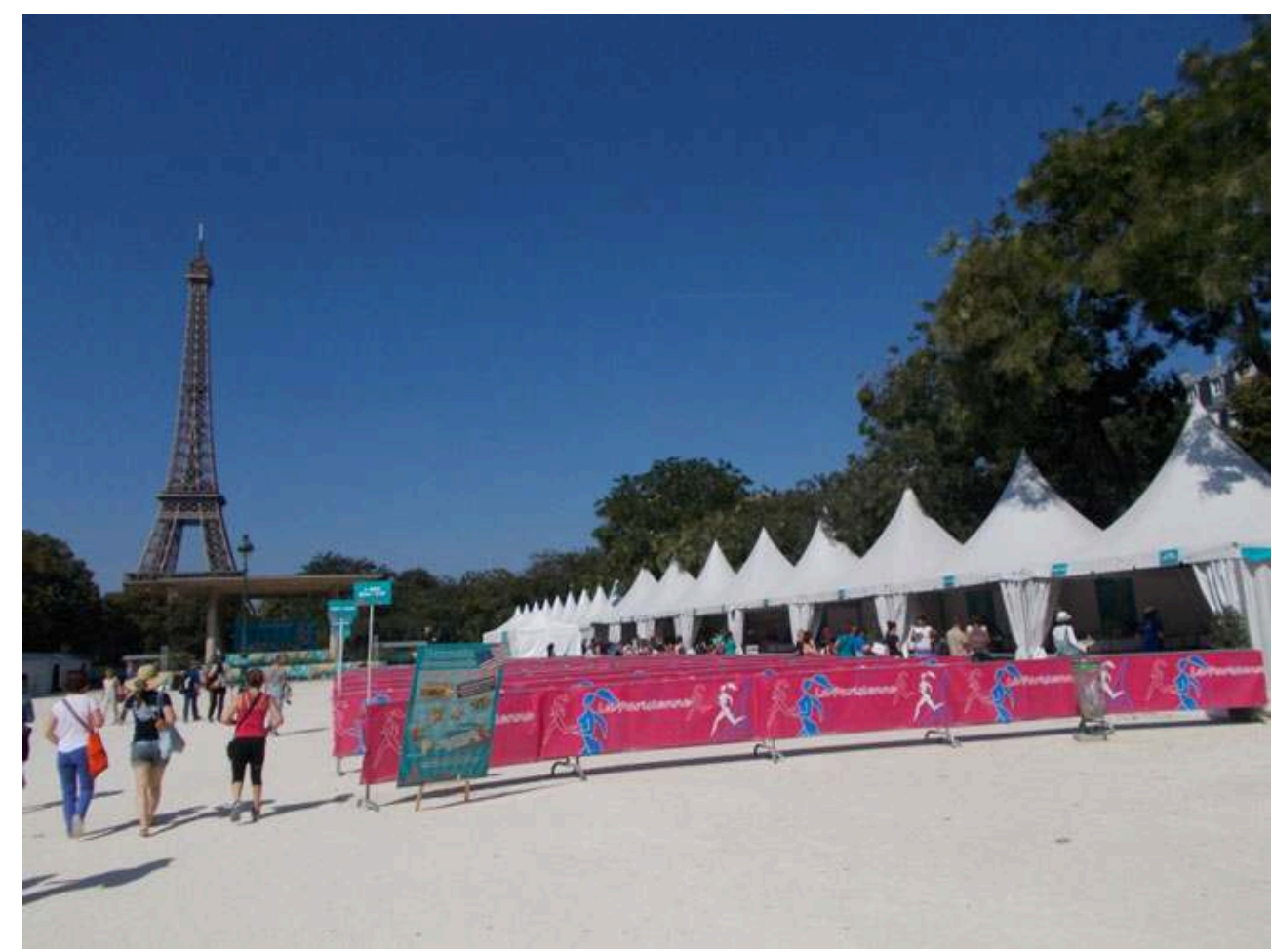

Bourbillères, 2014 
Figure 2. Les groupes d'entreprise et les particuliers se mélangent pendant la course sous le regard de centaines de spectateurs

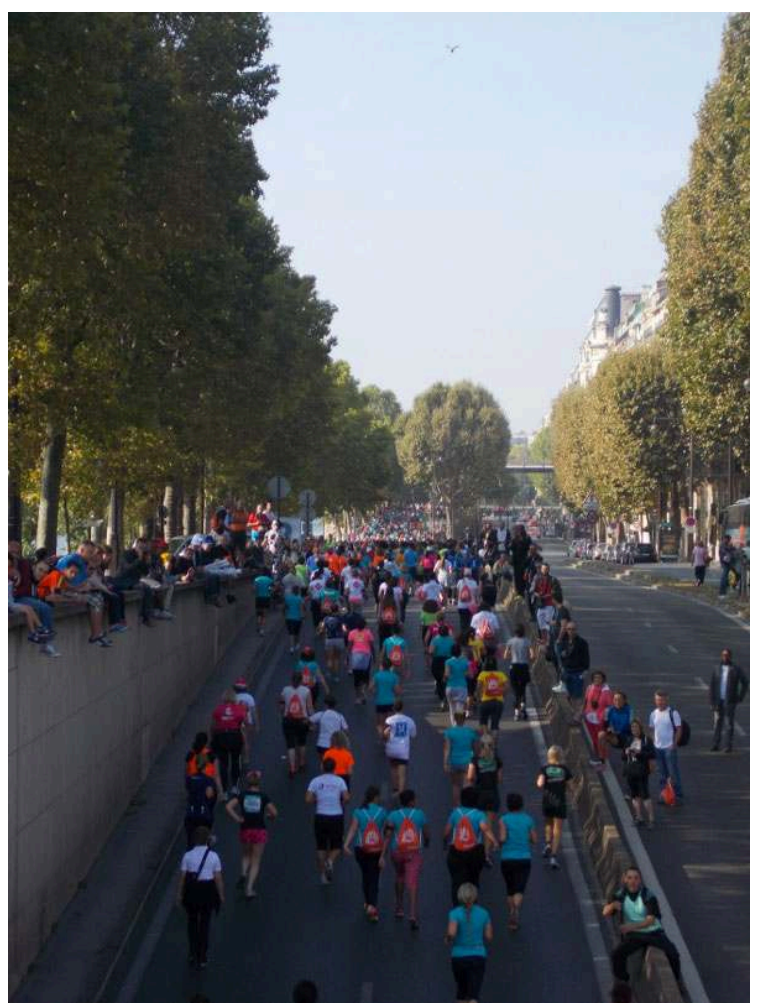

Bourbillères, 2014

$5 \quad$ Le bruit et le son sont des objets géographiques (Montès, 2003). Ici, cela se formalise par des échauffements en musique, des activités de zumba ou de gymnastique suédoise qui participent de l'atmosphère. La danse est encouragée au moment du départ de chacune des vagues de participantes ou lors des animations dans le « village partenaire » devant l'École militaire. Pendant la course, de nombreux groupes de musique répartis sur le parcours contribuent à la création d'une ambiance joviale. De son côté, le speaker, depuis la ligne d'arrivée, rythme la matinée par son discours sur les partenaires commerciaux. Le marquage sonore est un élément révélateur du positionnement de l'événement, ici, la dimension promotionnelle et commerciale transparaît.

6 La ville dispose d'un patrimoine sonore multiple, support de politiques urbaines, qui participe à la création de sa symbolique et de son identité (Montès, 2003). Dans ce cas, la ville de Paris en soutenant le projet événementiel ${ }^{2}$ se sert de ses attributs (allant du nom et du logo jusqu'aux photos sous la tour Eiffel) pour alimenter sa communication territoriale ${ }^{3}$. 
Figure 3. Les vagues de départ de milliers de joggeuses au pied de la tour Eiffel pendant les échauffements en musique

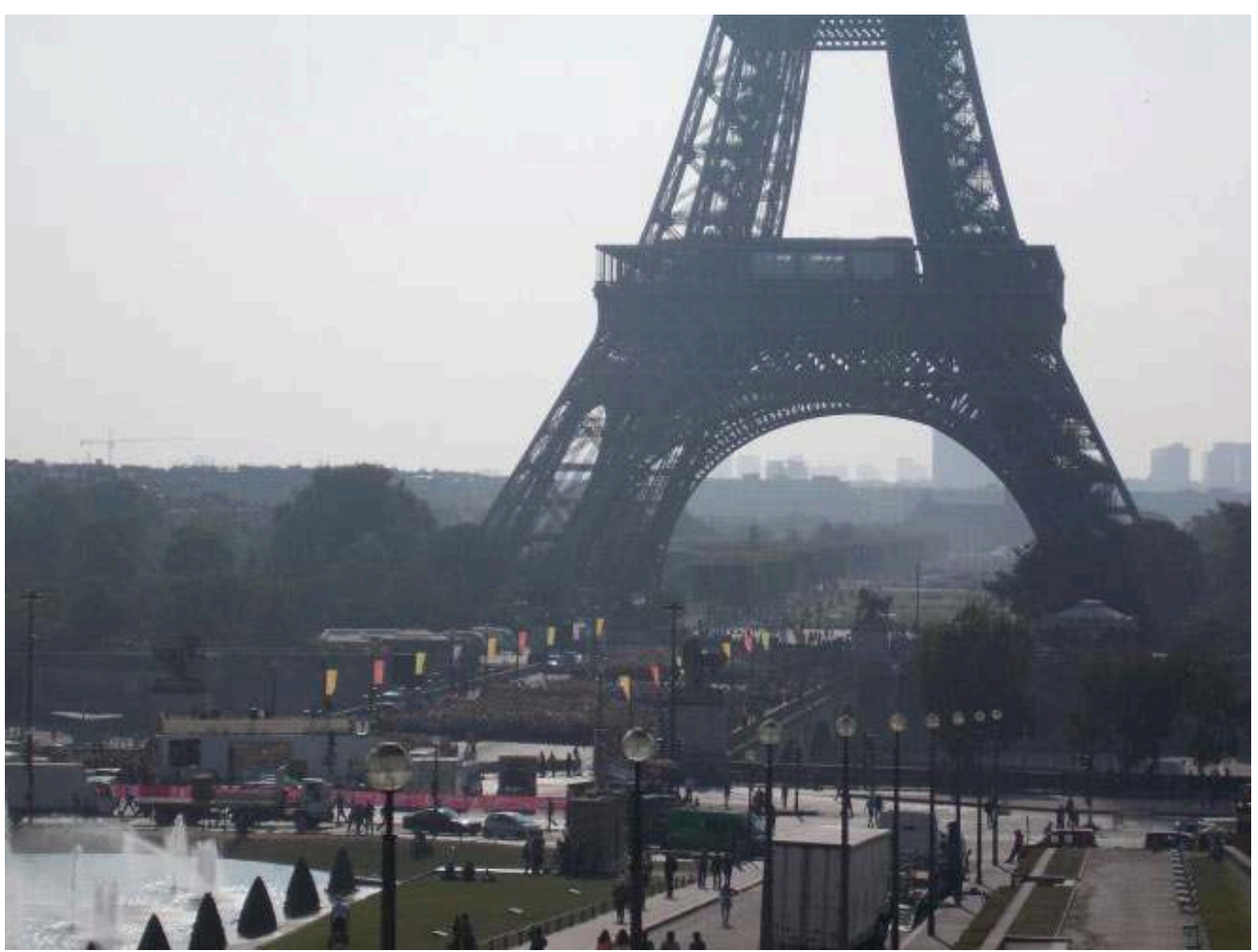

\section{Roland Garros dans la ville : une domination symbolique de la $\mathrm{FFT}^{4}$}

7 Roland Garros est le tournoi de tennis professionnel créé en 1925 dont la particularité est de se pratiquer sur terre battue. Il se déroule à Paris dans le stade Roland Garros depuis 1928, la dernière semaine de mai et la première semaine de juin. Il est l'événement tennistique qui bénéficie de la plus large audience ou couverture médiatique à travers la planète (Pulman, 2013). Pourtant, à l'échelle parisienne l'événement parait «fermé » en raison de son mode d'organisation et son public assez sélectif : culture tennistique, prix des places, communication « prestige » (Bourbillères, 2017). Par son importance médiatique, les flux économiques qu'il génère et sa volonté d'entretenir de bonnes relations avec la ville de Paris, il est toutefois dans l'obligation de se doter d'un dispositif accessible au grand public.

L'opération «Roland Garros dans la ville» permet de faire sortir le tournoi de l'enceinte du stade pour aller à la rencontre des Parisiens (terrains, écran géant, animations partenaires gratuites). De manière générale, le marquage urbain qui est associé à l'événement est minimal, mais particulièrement symbolique en s'établissant sur des éléments patrimoniaux forts de la capitale. 
Figure 4. Balle de tennis géante accrochée à la Tour Eiffel, liant symboliquement l'image du tournoi et celle de la ville de Paris

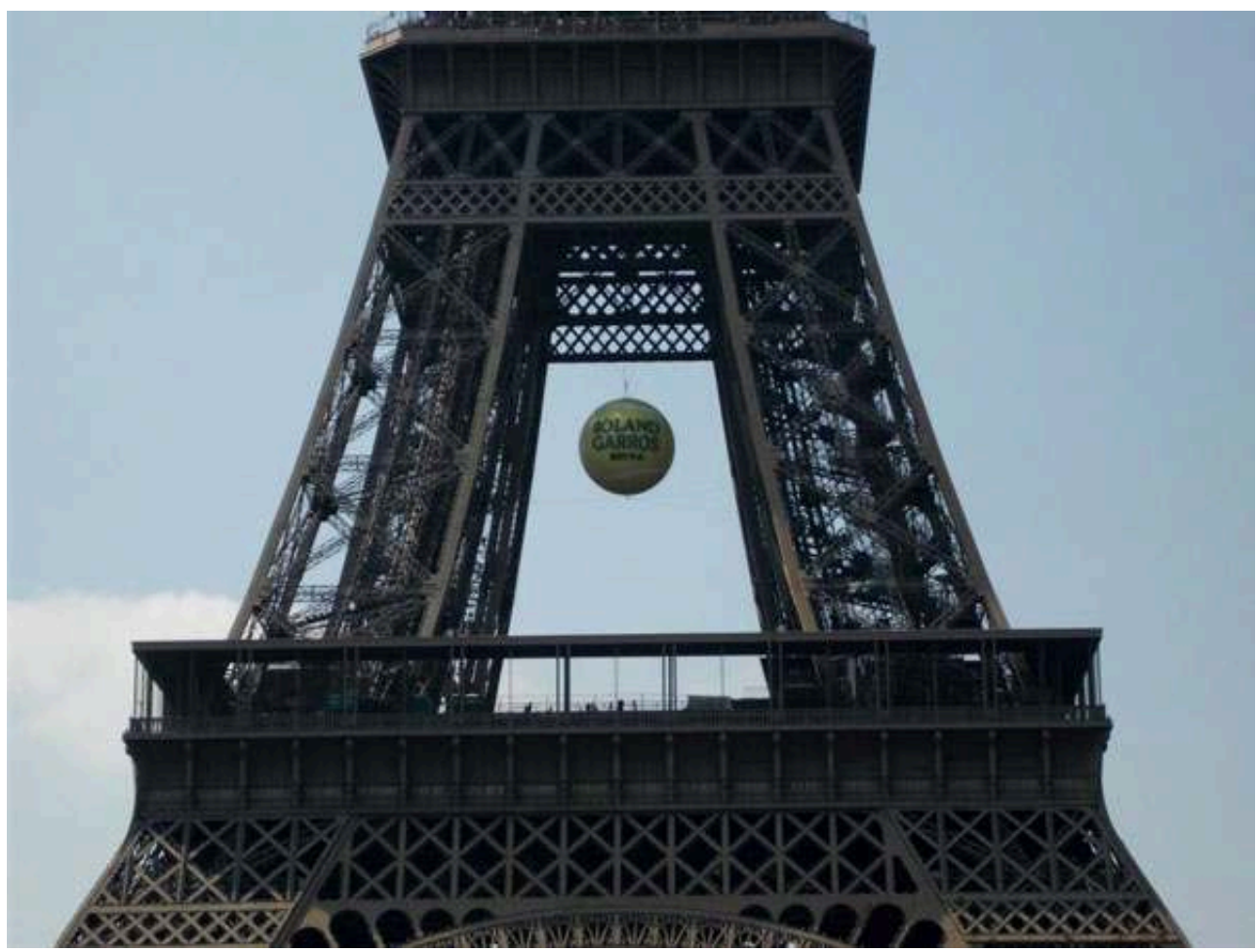

Bourbillères, 2014

La balle de tennis géante accrochée au premier niveau de la structure de la tour Eiffel pendant la quinzaine est un attribut assurant la réussite des images d'hélicoptères et des cartes postales. C'est donc un double objectif, interne à la ville de Paris pour créer de l'émulation autour de l'événement et externe, à l'international, pour alimenter son rayonnement et garantir « ce dans quoi les autres nous reconnaissent » (Akoun, Ansart, 1999). Ces attributs qui utilisent des repères signifiants (monuments, places) ou des corps et des signes mouvants (habits, pancartes) «marquent» les esprits et associent par l'événement sportif un lieu à des groupes sociaux (Veschambre, 2005). Cavaillé (1999) explique que «notre identité sociale apparaît toujours en premier lieu dans et par l'espace ». Dès lors, le marquage urbain représente une forme de matérialisation de l'identité, à la fois individuelle et collective (Veschambre, 2000). À cet égard, il devient aussi un indicateur de lecture du politique par la recherche des organisateurs à se démarquer dans l'espace social.

Dans ce cas, le marquage est produit par l'association symbolique entre un édifice "patrimonialisé » (tour Eiffel) et une institution (FFT) qui se l'approprie par une intervention matérielle. Cette visibilité sociale dans la dimension spatiale traduit une forme de pouvoir (Verret, 1995). En 1993, Bourdieu insiste sur l'intérêt de décrypter ce marquage symbolique, en l'inscrivant dans des rapports de force : « l'espace est un des lieux où le pouvoir s'affirme et s'exerce, et est sans doute sous la forme la plus subtile, celle de la violence symbolique, comme violence inaperçue " (Bourdieu, 1993). Les cas de Roland Garros et de «La Parisienne » révèleraient donc une forme de domination symbolique dans l'espace public en s'établissant sur le Champ-de-Mars. 
11 Un autre exemple marquant a été l'organisation de la Fan Zone parisienne pendant l'Euro 2016. La ville de Paris a accompagné le projet événementiel, allant même jusqu'à " privatiser $^{5}$ » le Champ-de-Mars. La ville espérait en retirer un bénéfice en termes d'image et de notoriété, quitte à mettre en scène des opérateurs privés en leur consacrant une forme d'hégémonie symbolique dans l'espace social.

\section{Le Champ-de-Mars : du support de communication à la ressource territoriale}

12 À Paris, les marquages urbains semblent être révélateurs des choix en termes de politiques sportives. La ville accompagne le développement de grands événements sportifs pour asseoir l'image d'une capitale dynamique et prestigieuse en s'appuyant sur des symboles inhérents au territoire. Par exemple, Roland Garros et « La Parisienne ", à travers leurs attributs visuels et sonores, traduisent un positionnement très clair et cohérent : des visuels (sobres et discrets pour l'un, colorés pour l'autre), une sémiotique patrimoniale (Champ-de-Mars et tour Eiffel mobilisés), un environnement sonore singulier (silencieux et respectable pour l'un, musical et diversifié pour l'autre) et des logos évocateurs. Ces attributs peuvent traduire une forme de consubstantialité (Bessy, 2013) entre l'événement et le territoire par la création d'une image commune : un Paris prestigieux (Roland Garros) et dynamique (« La Parisienne »).

13 Certains bâtiments des premières villes "touristiques » sont devenus emblématiques, et ont été étroitement associés à leur territoire. Reproduits sur des supports différents (cartes postales, affiches, dépliants publicitaires, etc.), ils sont devenus des emblèmes territoriaux, car ils ont « imagé » le territoire (Fagnoni, 2015). À Paris, c'est bien sûr le cas du Champ-de-Mars. Le patrimoine appréhendé comme ressource territoriale (Gumuchian, Pecqueur, 2007) renvoie également à la notion de « haut lieu ", ce qui pose la question de l'attractivité, généralement considérée comme un facteur de vitalité du territoire (Fagnoni, 2013). Reste à savoir si ces événements sportifs en sont réellement un vecteur, au-delà de la supposée image véhiculée.

\section{BIBLIOGRAPHIE}

ANSART P., AKOUN A. éd. (1999). Dictionnaire de sociologie. Paris : Le Robert Seuil.

BESSY O. (2013). « De l'innovation événementielle à l'innovation territoriale ». In Bessy O. (dir.), L'Innovation dans l'événementiel sportif. De l'attractivité touristique au développement des territoires. PUS, p. 38-53.

BOURBILLÈRES H. (2017). Impacts territoriaux de neufévénements sportifs parisiens (2013-2016). L'approche par les dynamiques locales. Thèse de doctorat. Université Paris Sud/Paris Saclay.

BOURDIEU P., ACCARDO A., BALAZS G., BEAUD S. (1993). La misère du monde. Paris : Seuil. 
CAVAILLÉ F. (1999). L'expérience de l'expropriation. ADEF

FAGNONI É. éd. (2013). La ressource territoriale entre patrimoine et création. Bulletin de l'Association de Géographes français.

FAGNONI É., BONNET-PINEAU É., GRAVARI-BARBAS M. (2015). « Patrimoine et patrimonialisation, de l'objet à la relation ». Compte rendu du Café Géographique du 24 novembre 2015 à Paris (Café de Flore). Les Cafés Géographiques. En ligne : http://cafe-geo.net/patrimoine-et-patrimonialisation-de-lobjeta-la-relation/

GUMUCHIAN H., PECQUEUR B., dir. (2007). La ressource territoriale. Paris : Economica, $252 \mathrm{p}$.

ISBN 2-7178-5253-0

MONTÈs C. (2003). « La ville, le bruit et le son, entre mesure policière et identités urbaines ». Géocarrefour, 78(2), p. 91-94. En ligne : https://journals.openedition.org/geocarrefour/168 PULMAN B. (2013). Rouge est la terre. Paris : Calmann-Lévy. 304 p. ISBN 9782702153598

VERRET M. (1995). Chevilles ouvrières. Paris : Les Éditions de l'Atelier/Éditions ouvrières, coll. « Mouvement social », 254 p. ISBN 9782708231177

VESCHAMBRE V. (2007). « Patrimoine : un objet révélateur des évolutions de la géographie et de sa place dans les sciences sociales ». Annales de géographie, vol. 4, nº 656, p. 361-381.

VESCHAMBRE V. (2005). « Le recyclage urbain, entre démolition et patrimonialisation : enjeux d'appropriation symbolique de l'espace ». Norois, n 195, p. 79-92.

VESCHAMBRE V. (2000). « Patrimonialisation et enjeux politiques : les édifices Le Corbusier à Firminy ». In « Patrimoine et environnement : les territoires du conflit ». Norois, $\mathrm{n}^{\circ} 185$, p. $125-138$.

\section{NOTES}

1. Le 14 juillet 1790, eut lieu la fête de la Fédération. Le 17 juillet 1791, s'y produisit la fusillade du Champ-de-Mars. Jean Sylvain Bailly y fut guillotiné le 12 novembre 1793. On y célébra la fête de l'Être suprême, le 8 juin 1794. En 1815, Napoléon y passe en revue toute sa Garde ainsi que 60000 hommes de la garde nationale de Paris. Le 8 janvier 1887, le choix de construire la Tour Eiffel sur ce site est entériné.

2. Communication, subventions les premières années de 1997 à 2001, autorisations administratives, support logistique.

3. Les élus de la ville, la maire Anne Hidalgo ou l'adjoint au sport et au tourisme JeanFrançois Martins, tiennent un discours mélioratif sur ce projet régulièrement cité en exemple pour souligner le dynamisme événementiel de la ville. Le « développement du sport féminin » est aussi un argument politiquement porteur car il fait l'objet d'un consensus général autour de la nécessité de le mettre à l'agenda public.

4. Fédération Française de Tennis.

5. Au sens d'en restreindre l'accès. 


\section{AUTEURS}

\section{HUGO BOURBILLÈRES}

Post-doctorant au laboratoire Sport et Sciences Sociales (EA 1342), Université de Strasbourg

BARBARA EVRARD

Maitre de conférences, Normandie Université, Laboratoire CETAPS EA 3832

DOMINIQUE CHARRIER

Maitre de conférences HDR, Laboratoire CIAMS EA 4532, Université Paris Sud 